\title{
Right Gluteus Medius
}

National Cancer Institute

\section{Source}

National Cancer Institute. Right Gluteus Medius. NCI Thesaurus. Code C105639.

One of the three gluteal muscles on the lateral surface of the pelvic bone that abducts and rotates the thigh located on the right side of the body. 